\title{
Diagnostic Value of Biopsy Techniques in Lumbar Spondylodiscitis: Percutaneous Needle Biopsy and Open Biopsy
}

\author{
Kyoung Hyup Nam', Geun Sung Song ${ }^{2}$, In Ho Han', \\ Byung Kwan Choi ${ }^{1}$, Seung Heon Cha ${ }^{1}$ \\ ${ }^{1}$ Department of Neurosurgery, Pusan National University Hospital, Pusan National University School of Medicine, Busan, \\ ${ }^{2}$ Department of Neurosurgery, Pusan National University Yangsan Hospital, Pusan National University \\ School of Medicine, Yangsan, Korea
}

Objective: The objective of this study was to evaluate and compare the diagnostic value of the open biopsy technique and the percutaneous biopsy techniques in lumbar spondylodiscitis.

Methods: Between January 2004 and December 2009, we retrospectively reviewed the medical records of 57 patients with infectious lumbar spondylodiscitis. The etiologic diagnosis of the infectious spondylodiscitis was obtained by two methods. Of 57 cases, twenty-seven patients underwent open biopsy and thirty patients underwent percutaneous needle biopsy including computed tomography (CT) - guided and fluoroscopy-guided needle aspiration. All biopsies were performed by experienced two neurosurgeons and one interventional radiologist.

Results: Of the 57 cases radiologically consistent with spinal infection, 29 (50.9\%) biopsy specimens resulted in positive cultures and $28(49.1 \%)$ returned negative cultures. According to the type of biopsy techniques, the culture-positive rate was higher $(p=0.005)$ in the open biopsy group than the percutaneous needle biopsy group. $19(70.4 \%)$ of 27 biopsy specimens were positive in the open biopsy group, and $10(33.3 \%)$ of 30 biopsy specimens were positive in the percutaneous needle biopsy group. Furthermore, the open biopsy showed higher positive culture rate than the percutaneous needle biopsy in cases with administration of empirical antibiotics although there was no statistically significant $(p=0.137)$.

Conclusions: Open biopsy should be considered for administration of organism-specific antibiotics for the successful treatment when percutaneous needle yield negative result. Furthermore, empirical antibiotics should be delayed until results of cultures unless the patient is severely septic, critically ill, neutropenic or neurologically compromised.

Key Words: Diagnostic Techniques $\cdot$ Biopsy $\cdot$ Surgical $\cdot$ Needle $\cdot$ Spondylodiscitis

\section{INTRODUCTION}

Infectious spondylodiscitis is common inflammatory disorder and serious condition that can occur spontaneously or postoperatively at vertebral body, intervertebral disc, spinal canal and paravertebral soft tissue ${ }^{4,16}$. However, infectious spondylodiscitis is often difficult to diagnose and treat. Infectious spondylodiscitis is characterized clinically by relatively non-specific symptoms such as fever and axial pain. Furthermore, some cases of infectious spondylodiscitis may appear

\footnotetext{
- Received: December 13, 2011 - Revised: December 28, 2011

- Accepted: January 13, 2012

Corresponding Author: Geun Sung Song, MD, PhD

Department of Neurosurgery, Pusan National University Yangsan

Hospital, Beomeo-ri, Mulgeum-eup, Yangsan 626-770, Korea

Tel: +82-55-360-2126, Fax: +82-55-360-2156

E-mail: gnsong@pusan.ac.kr
}

similar to metastatic lesions in $\mathrm{MRI}^{12)}$.

Surgical treatment is usually reserved for patients with failed antibiotics therapy, progressive spinal deformity or instability, epidural abscess, or neurological impairment ${ }^{14,19,24,25)}$. Thus, in treatment of infectious spondylodiscitis, early identification of the etiologic organism is very important to administrate organism-specific antibiotics for the successful treatment ${ }^{3,4,20)}$.

Recently, various percutaneous biopsy techniques for bacteriologic diagnosis have been performed as minimally invasive methods. Keeping up with the development of percutaneous biopsy techniques, the percutaneous biopsy has been performed more commonly than open biopsy. However, percutaneous spinal biopsy has a variable rate of success in infectious spondylodiscitis $^{10,13,17,19,23,25)}$.

The purpose of our study was to evaluate and compare the diagnostic value of the open biopsy technique and the percutaneous biopsy techniques in lumbar spondylodiscitis. 


\section{MATERIAL AND METHODS}

We retrospectively reviewed the medical records of $57 \mathrm{pa}$ tients with infectious lumbar spondylodiscitis between January 2004 and December 2009. There were 33 men (57.9\%) and 24 women (42.1\%) with a mean age of 61.6 years (range, 12-82 years). Infectious spondylodiscitis was diagnosed based on radiographic finding and clinical examinations, including elevated erythrocyte sedimentation rate and C-reactive protein values. Of the 57 patients, infectious spondylodiscitis occurred spontaneously in 42 cases $(73.7 \%)$ and postoperatively in 15 cases (26.3\%). Empirical antibiotics were administrated before biopsy procedures in 27 cases (47.4\%). There were no significant demographic differences between the two groups (Table 1).

The etiologic diagnosis of the infectious spondylodiscitis was obtained by two methods. Of the 57 cases, twenty-seven patients underwent open biopsy and thirty patients underwent percutaneous needle biopsy including computed tomography (CT) - guided and fluoroscopy - guided needle aspiration. All biopsies were performed by experienced two neurosurgeons and one interventional radiologist.

Microbiologic analysis consisted of various combinations of cultures including aerobic, anaerobic, fungus, and mycobacterium with Gram stain, fungal stain and acid-fast stain. A positive microbiologic result was defined as the demonstration of a pathogenic organism, either by growth in culture or by microscopic stain. Radiographic finding, clinical man-

Table 1. Summary of characteristics in 57 cases of spondylodiscitis

\begin{tabular}{lccc}
\hline \hline & \multicolumn{3}{c}{ Type of methods } \\
\cline { 2 - 4 } & $\begin{array}{c}\text { Surgical Open Percutaneous } \\
\text { biopsy }\end{array}$ & $\begin{array}{c}\text { Needle biopsy } \\
\text { p-value }\end{array}$ \\
\hline Mean Age (yrs) & 59.1 & 64.0 & \\
SEX & & 18 & \\
M & 15 & 12 & \\
F & 12 & & 0.200 \\
Cause of spondylodiscitis & & 20 & \\
Spontaneous infection & 22 & 10 & \\
$\begin{array}{l}\text { Postoperative infection } \\
\text { Administration of Empiri- }\end{array}$ & 5 & & 0.182 \\
cal antibiotics prior to & & & \\
procedure & & 12 & \\
Yes & 15 & 18 & \\
No & 12 & & \\
\hline
\end{tabular}

Statistical analyses by Fisher's exact test agement and outcome were not part of our study.

Statistical analyses were performed using SPSS statistic software $(12.0 \mathrm{~K})$. Fisher's exact test was used to assess the culture positive rate. A $p$ value of less than 0.05 was considered statistically significant.

\section{RESULTS}

Of the 57 cases radiologically consistent with spinal infection, 29 (50.9\%) biopsy specimens resulted in positive cultures and $28(49.1 \%)$ returned negative cultures. The various causative pathogens diagnosed on culture are shown in Table 2. Causative pathogens were identified in positive biopsy cultures were Staphylococcus aureus (13), Mycobacterium tuberculosis (9), Pseudomonas aeruguinosa (3), Candida albicans (1), Enterobacter cloacae (1), Klebsiella pneumonia (1), and Streptococcus spp (1). All of Staphylococcus aureus were methiciline-resistant staphylococcus aureus (MRSA).

We evaluated the culture-positive rates according to the type of biopsy techniques and administration of empirical antibiotics prior to procedures. According to the type of biopsy techniques, the culture-positive rate was higher $(\mathrm{p}=0.005)$ in the open biopsy group than the percutaneous needle biopsy group. 19 (70.4\%) of 27 biopsy specimens were positive in the open biopsy group, and $10(33.3 \%)$ of 30 biopsy specimens were positive in the percutaneous needle biopsy group (Table 3).

There was no significant difference in administration of empirical antibiotics prior to procedures between two groups (Table 1). Regardless of procedures, 11 (40.7\%) of 27 cases were positive in antibiotics administration group and 18 (60.0\%) of 30 cases were positive in no administration group $(\mathrm{p}=$ 0.182). However, culture-positive rate was higher $(\mathrm{p}=0.005)$ in the open biopsy group among 24 cases without administration of empirical antibiotics before procedures; 11 (91.7\%) of 12 biopsy specimens were positive in the open biopsy group and $7(58.3 \%)$ of 12 biopsy specimens were positive in the

Table 2. Summary of causative microorganism

\begin{tabular}{lccc}
\hline \hline Offending pathogens & Open $\mathrm{Bx}$ & Percutaneous $\mathrm{Bx}$ & Total \\
\hline Staphylococcus & 7 & 6 & 13 \\
Mycobacterium & 6 & 3 & 9 \\
Pseudomonas & 2 & 1 & 3 \\
Candida & 1 & 0 & 1 \\
Enterobacter & 1 & 0 & 1 \\
Klebsiella & 1 & 0 & 1 \\
Streptococcus & 1 & 0 & 1 \\
Culture negative & 8 & 20 & 28 \\
\hline Total & 27 & 30 & 57 \\
\hline
\end{tabular}


Table 3. Culture positive rate according to types of biopsy technique

\begin{tabular}{lccc}
\hline \hline & \multicolumn{3}{c}{ Type of methods } \\
\cline { 2 - 4 } Result of culture & $\begin{array}{c}\text { Microsurgical } \\
\text { Open Biopsy (\%) }\end{array}$ & $\begin{array}{c}\text { Percutaneous } \\
\text { Peedle Biopsy (\%) }\end{array}$ & p-value \\
\hline Positive & $19(70.4)$ & $10(33.3)$ & 0.005 \\
Negative & $8(29.6)$ & $20(66.7)$ & \\
\hline
\end{tabular}

Statistical analyses by Fisher's exact test

Table 4. Correlation between culture positive rate and empirical antibiotics

\begin{tabular}{|c|c|c|c|c|}
\hline & \multicolumn{4}{|c|}{ No. of patient (\%) } \\
\hline & Positive & Negative & Total & $\mathrm{p}$-value \\
\hline $\begin{array}{l}\text { Administration of empirical } \\
\text { antibiotics }\end{array}$ & & & & 0.137 \\
\hline Microsurgical open biopsy & 8 & 7 & 15 & \\
\hline Percutaneous needle biopsy & 3 & 9 & 12 & \\
\hline $\begin{array}{l}\text { No Administration of empi- } \\
\text { rical antibiotics }\end{array}$ & & & & 0.005 \\
\hline Microsurgical open biopsy & 11 & 1 & 12 & \\
\hline Percutaneous needle biopsy & 7 & 11 & 12 & \\
\hline
\end{tabular}

percutaneous needle biopsy group (Table 4). Furthermore, the open biopsy showed higher positive culture rate than the percutaneous needle biopsy in cases with administration of empirical antibiotics although there was no statistically significant (open biopsy 53.3\%, percutaneous needle biopsy 25.0\%; $\mathrm{p}=$ 0.137) (Table 4).

Any complication or side effect related to procedures did not occur in either group during follow-up.

\section{DISCUSSION}

Infectious spondylodiscitis is common inflammatory disorder and serious condition that can occur spontaneously or postoperatively at vertebral body, intervertebral disc, spinal canal and paravertebral soft tissue ${ }^{4,16}$. Keeping up with the advance in radiologic technique, and laboratory test, diagnosis of spinal infection has been developed to avoid the severely debilitating complications of delayed or inadequate treatment $^{4,20,24)}$. However, infectious spondylodiscitis is often difficult to diagnose and treat because infectious spondylodiscitis is characterized clinically by relatively non-specific symptoms such as fever and axial pain. Furthermore, some cases of infectious spondylodiscitis may appear similar to metastatic lesions in magnetic resonance images ${ }^{12}$.
Surgical treatment is usually reserved for patients with failed antibiotics therapy, progressive spinal deformity or instability, epidural abscess, or neurological impairment ${ }^{14,19,24,25)}$. Thus, in treatment of infectious spondylodiscitis, early identification of the etiologic organism is very important to administrate organism-specific antibiotics for the successful treatment ${ }^{3,4,20)}$. When clinical history, laboratory test, and radiologic finding suggest infectious spondylodiscitis, infectious tissue must be obtained to identify the etiologic organism.

Recently various percutaneous biopsy techniques for bacteriologic diagnosis have been performed as minimally invasive methods such as CT-guided and fluoroscopy-guided needle biopsy. Since needle biopsy of the spine was first reported by Ball in 1934, the percentages of successful closed needle biopsies have been increased and percutaneous needle biopsy has been shown to be safe and effective diagnostic modality in the spondylodiscitis ${ }^{1,5,18}$. However, previously reported series of imaging-guided needle biopsy in the spinal infection have been variable in their yield of microbiologically identified pathogens. Percutaneous needle biopsy techniques have a variable rate of success from $36 \%$ to $91 \%$ in the infectious spondylodiscitis ${ }^{5,718,22)}$. Chew and Kline reported that CT-guided percutaneous aspiration specimens were positive rate as $91 \% \%^{5)}$. Staatz et al reported $76 \%$ culture-positive rate using CT-guided biopsy, Perronne et al reported a $74 \%$ culture-positive rate by the uoroscopy-guided needle biopsy, and Fouquet et al obtained bacteriological diagnosis in 36\% biopsies using a Mazabraud trocar ${ }^{7,18,22)}$.

Since Adapon et al. first described CT-guided percutaneous needle biopsy, spine biopsy using CT guidance has been used widely as cost-effective procedure ${ }^{1)}$. CT-guided biopsy can make accurate targeting safely to various target tissue. Chew and Kline reported that CT-guided biopsy is an accurate method for identifying pathogens in active bacterial disc space infections, but is less reliable for identifying fungal infections ${ }^{5}$. Although the result of percutaneous needle biopsy can be false negative for various reasons, a second percutaneous biopsy should be conducted if the initial cultures are non-diagnostic ${ }^{8)}$. If the second percutaneous biopsy is also negative, an open biopsy should be considered.

Intraoperative removal of tissue specimens by open biopsy is the most reliable method of detecting the pathogen in lumbar spondylodiscitis ${ }^{21}$. An open biopsy may be considered when a repeat percutaneous biopsy technique is culture negative and there is no improvement on empirical antibiotics, when the infection is inaccessible to percutaneous techniques, and in the presence of marked structural damage or neurological de?cit ${ }^{6,9)}$. Open biopsy provides culture-positive rate in more than $75 \%$ of cases ${ }^{2,6,15)}$.

In our study, the culture-positive rate of percutaneous nee- 
dle biopsy was $33.3 \%$ (10 of 30 cases) that relatively lower than other reports. The result of percutaneous needle biopsy can be false negative for various reasons, including an inadequate amount of specimen, sampling error, and empirical antibiotics at the time of the biopsy ${ }^{19,24)}$. In addition to these cause, the result may depend on experience and skill of radiologist and sometimes difficulty of paraspinal approach into disc. Hadjipavlou and colleagues argued that the transpedicular biopsy allows for larger bony sampled to be obtained and yields better results than traditional percutaneous needle biopsy technique $^{11)}$. The transpedicular approach is safely performed with either CT or fluoroscopy.

In the open biopsy group, culture-positive rate was similar with previous reports as $70.4 \%$ (19 of 27 cases). Furthermore, the culture-positive rate of open biopsy was much higher than the percutaneous needle biopsy in patients who are not being treated with empirical antibiotics at the time of the biopsy. In patients who were administrated the empirical antibiotics prior to procedures, the culture-positive rate was lower and there was no significant differences in two biopsy methods. Rankine et al reported that the administration of empirical antibiotics before biopsy reduces the chance of isolating an organism and determining antibiotic sensitivity ${ }^{19}$.

\section{CONCLUSION}

Percutaneous biopsy techniques are safe and minimally invasive diagnostic procedures to identify pathogens in lumbar spondylodiscitis. However, open biopsy provided much higher accuracy than percutaneous needle biopsy for lumbar infectious spondylodiscitis. We concluded that open biopsy should be considered for administration of organism-specific antibiotics for the successful treatment when percutaneous needle yield negative result. Furthermore, empirical antibiotics should be delayed until results of cultures unless the patient is severely septic, critically ill, neutropenic or neurologically compromised.

\section{REFERENCES}

1. Adapon BD, Legada BD, Jr., Lim EV, Silao JV, Jr., DalmacioCruz A: CT-guided closed biopsy of the spine. J Comput Assist Tomogr 5:73-78, 1981

2. An H, Seldomridge J: Spinal Infections. Clin Orthop Relat Res 444:27-33, 2006

3. Asamoto S, Doi H, Kobayashi N, Endoh T, Sakagawa H, Iwanaga Y, et al: Spondylodiscitis: diagnosis and treatment. Surgical Neurology 64:103-108, 2005

4. Bonfiglio M, Lange T, Kim Y: Pyogenic vertebral osteomye- litis. Disk space infections. Clin Orthop Relat Res 96:234247, 1973

5. Chew FS, Kline MJ: Diagnostic yield of CT-guided percutaneous aspiration procedures in suspected spontaneous infectious diskitis. Radiology 218:211-214, 2001

6. Cottle L, Riordan T: Infectious spondylodiscitis. Journal of Infection 56:401-412, 2008

7. Fouquet B, Goupille P, Gobert F, Cotty P, Roulot B, Valat JP: Infectious discitis diagnostic contribution of laboratory tests and percutaneous discovertebral biopsy. Rev Rhum Engl Ed 63:24-29, 1996

8. Friedman RL: A comparison of accuracy between clinical examination and magnetic resonance imaging in the diagnosis of meniscal and anterior cruciate ligament tears. Arthroscopy 13:271-275; author reply 276-277, 279-280, 1997

9. Govender S: Spinal infections. J Bone Joint Surg Br 87:14541458, 2005

10. Haaker RG, Senkal M, Kielich T, Kramer J: Percutaneous lumbar discectomy in the treatment of lumbar discitis. European Spine Journal 6:98-101, 1997

11. Hadjipavlou AG, Katonis PK, Gaitanis IN, Muffoletto AJ, Tzermiadianos MN, Crow W: Percutaneous transpedicular discectomy and drainage in pyogenic spondylodiscitis. European Spine Journal 13:707-713, 2004

12. Hsu CY, Yu CW, Wu MZ, Chen BB, Huang KM, Shih TT: Unusual manifestations of vertebral osteomyelitis: intraosseous lesions mimicking metastases. AJNR Am J Neuroradiol 29:1104-1110, 2008

13. Ito M, Abumi K, Kotani Y, Kadoya K, Minami A: Clinical outcome of posterolateral endoscopic surgery for pyogenic spondylodiscitis: results of 15 patients with serious comorbid conditions. Spine (Phila Pa 1976) 32:200-206, 2007

14. Krodel A, Sturz H, Siebert CH: Indications for and Results of Operative Treatment of Spondylitis and Spondylodiscitis. Archives of Orthopaedic and Trauma Surgery 110:78-82, 1991

15. Lam K, Webb J: Discitis. Hosp Med 65:280-286, 2004

16. Maiuri F, Iaconetta G, Gallicchio B, Manto A, Briganti F: Spondylodiscitis - Clinical and magnetic resonance diagnosis. Spine 22:1741-1746, 1997

17. Nolla JM, Ariza J, Gomez-Vaquero C, Fiter J, Bermejo J, Valverde J, et al: Spontaneous pyogenic vertebral osteomyelitis in nondrug users. Semin Arthritis Rheum 31:271-278, 2002

18. Perronne C, Saba J, Behloul Z, Salmon-Ceron D, Leport C, Vilde JL, et al: Pyogenic and tuberculous spondylodiskitis (vertebral osteomyelitis) in 80 adult patients. Clin Infect Dis 19: 746-750, 1994

19. Rankine JJ, Barron DA, Robinson P, Millner PA, Dickson RA: Therapeutic impact of percutaneous spinal biopsy in spinal infection. Postgrad Med J 80:607-609, 2004

20. Rezai AR, Woo HH, Errico TJ, Cooper PR: Contemporary management of spinal osteomyelitis. Neurosurgery 44:10181025, 1999

21. Sobottke R, Seifert H, Fatkenheuer G, Schmidt M, Gossmann A, Eysel P: Current Diagnosis and Treatment of Spondylodiscitis. Deutsches Arzteblatt International 105:181-I, 2008

22. Staatz G, Adam GB, Keulers P, Vorwerk D, Gunther RW: 
Spondylodiskitic abscesses: CT-guided percutaneous catheter drainage. Radiology 208:363-367, 1998

23. Vinicoff PG, Gutschik E, Hansen SE, Karle A, Rieneck K: [CT-guided spinal biopsy in spondylodiscitis]. Ugeskr Laeger 160:5931-5934, 1998

24. Yang SC, Fu TS, Chen LH, Chen WJ, Tu YK: Identifying pathogens of spondylodiscitis: percutaneous endoscopy or CTguided biopsy. Clin Orthop Relat Res 466:3086-3092, 2008

25. Yang SC, Fu TS, Chen LH, Niu CC, Lai PL, Chen WJ: Percutaneous endoscopic discectomy and drainage for infectious spondylitis. Int Orthop 31:367-373, 2007 